Article

\title{
Associations between LncRNA MALAT1 Polymorphisms and Lymph Node Metastasis in Prostate Cancer
}

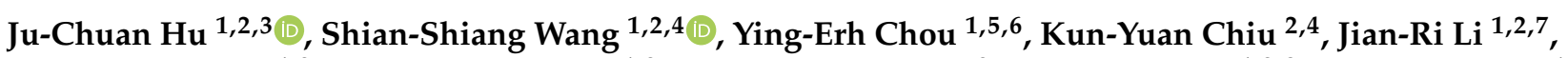 \\ Chuan-Shu Chen ${ }^{1,2}$, Sheng-Chun Hung ${ }^{1,2}$, Cheng-Kuang Yang ${ }^{2}$, Yen-Chuan Ou ${ }^{1,2,8}$, Chen-Li Cheng ${ }^{1,2}$, \\ Chia-Yen Lin $1,2, *(1)$ and Shun-Fa Yang $1,6, *$ (i)
}

1 Institute of Medicine, Chung Shan Medical University, Taichung 402, Taiwan; shelleycain525@gmail.com (J.-C.H.); sswdoc@yahoo.com.tw (S.-S.W.); intointo814@gmail.com (Y.-E.C.); fisherfishli@yahoo.com.tw (J.-R.L.); r2060d@yahoo.com.tw (C.-S.C.); weshong1118@gmail.com (S.-C.H.); ycou228@gmail.com (Y.-C.O.); cheng20011@gmail.com (C.-L.C.)

2 Division of Urology, Department of Surgery, Taichung Veterans General Hospital, Taichung 407, Taiwan; chiu37782002@yahoo.com (K.-Y.C.); yangck@vghtc.gov.tw (C.-K.Y.)

3 Division of Urology, Department of Surgery, Chiayi Branch, Taichung Veterans General Hospital, Chiayi 600, Taiwan

4 Department of Applied Chemistry, National Chi Nan University, Nantou 545, Taiwan

5 School of Medicine, Chung Shan Medical University, Taichung 402, Taiwan

6 Department of Medical Research, Chung Shan Medical University Hospital, Taichung 402, Taiwan

7 Department of Medicine and Nursing, Hung Kuang University, Taichung 433, Taiwan

8 Department of Urology, Tung's Taichung MetroHarbor Hospital, Taichung 433, Taiwan

* Correspondence: lcyhank.tw@gmail.com (C.-Y.L.); ysf@csmu.edu.tw (S.-F.Y.)

Citation: Hu, J.-C.; Wang, S.-S.; Chou, Y.-E.; Chiu, K.-Y.; Li, J.-R.; Chen, C.-S.; Hung, S.-C.; Yang, C.-K.; Ou, Y.-C.; Cheng, C.-L.; et al.

Associations between LncRNA MALAT1 Polymorphisms and Lymph Node Metastasis in Prostate Cancer. Diagnostics 2021, 11, 1692. https:// doi.org/10.3390/diagnostics11091692

Academic Editor: Nicola Longo

Received: 6 August 2021

Accepted: 14 September 2021

Published: 16 September 2021

Publisher's Note: MDPI stays neutral with regard to jurisdictional claims in published maps and institutional affiliations.

Copyright: (c) 2021 by the authors. Licensee MDPI, Basel, Switzerland. This article is an open access article distributed under the terms and conditions of the Creative Commons Attribution (CC BY) license (https:/ / creativecommons.org/licenses/by/ $4.0 /)$.

\begin{abstract}
Current evidence elucidates that long noncoding RNA metastasis-associated lung adenocarcinoma transcript 1 (MALAT1) could regulate genetic expression and play a crucial role in both the diagnosis and prognosis of prostate cancer. Single-nucleotide polymorphisms (SNPs) of MALAT1 could alter the oncogenesis in various cancers. However, the associations between MALAT1 SNPs and prostate cancer have barely been investigated to date. This study included 579 patients with prostate cancer who received robotic-assisted radical prostatectomy at Taichung Veterans General Hospital from 2012 to 2017. Three SNPs of MALAT1 were analyzed to identify the impacts of SNPs on the clinicopathologic features in Taiwanese prostate cancer. Our results show that patients with a polymorphic $\mathrm{G}$ allele at rs619586 had a significantly higher risk of being in an advanced Gleason grade group (AOR: 1.764; 95\% CI: 1.011-3.077; $p=0.046$ ). Moreover, individuals with at least one polymorphic A allele at MALAT1 rs1194338 in the PSA >10 ng/mL group were positively associated with node-positive prostate cancer. In conclusion, MALAT1 SNPs are significantly associated with the susceptibility to both advanced Gleason grade and nodal metastasis in prostate cancer. The presence of MALAT1 SNPs rs619586 and rs1194338 seems to enhance oncogenesis in prostate cancer.
\end{abstract}

Keywords: MALAT1; prostate cancer; polymorphism; metastasis

\section{Introduction}

In 2021, prostate cancer remains the most frequently diagnosed cancer and the second leading cause of mortality among American men [1]. Prostate-cancer-related death has diminished due to the extensive applications of prostate-specific antigen (PSA) testing; however, the overall downward trend of mortality stabilized after 2013, which is probably because of the recommendation of the United States Preventive Services Task Force (USPSTF) against PSA screening in 2012 and the rising diagnostic rates of late-stage prostate cancer [2]. There is a similar situation in Taiwan, although the overall incidence of prostate cancer is less frequent in Asia [3]. Less organ-confined prostate cancer and worse oncologic outcomes in the younger population have been reported in Taiwan [4,5]. Radical prostatectomy provides a definite treatment for prostate cancer, while concomitant pelvic lymph 
node dissection remains the gold standard for staging lymphadenopathy [6,7]. However, more aggressive operations come with the increased risk of surgical complications [8-10]. Therefore, more reliable prognostic predictors are still demanded in order to maintain the balance between overtreatment for prostate cancer and underestimation of cancer risk.

Tumorigenesis is an intricate process involving both genetic and epigenetic processes. For node-positive prostate cancer, both germline testing and somatic tumor testing for homologous recombination gene mutations (HRRm) and microsatellite instability (MSI) should be considered [11]. These molecular markers provide a precise guidance for further medical treatments, especially when prostate cancer progresses to castration-resistant status [12]. Long noncoding ribonucleic acids (lncRNAs) represent another potential diagnostic and therapeutic target in prostate cancer $[13,14]$. For example, prostate cancer antigen 3 (PCA3) gene testing used to enhance diagnostic accuracy is a well-known application of lncRNA in prostate cancer $[13,15,16]$.

Metastasis-associated lung adenocarcinoma transcript 1 (MALAT1), located on 11q13 in humans, is an lncRNA originally identified as a prognostic marker in non-small-cell lung cancer (NSCLC) [17]. Compared to PSA screening in prostate cancer, the urinary MALAT1 level is a more accurate diagnostic marker and helps to prevent $30.2-46.5 \%$ of unnecessary biopsies without missing any high-grade cancer in populations with PSA $4-10 \mathrm{ng} / \mathrm{mL}$ [18]. In addition to the diagnostic value, recent evidence indicates that MALAT1 could also serve as a therapeutic target in prostate cancer [17]. MALAT1 plays an essential role in the axis of the androgen receptor splicing variant 7 (AR-v7) and is found highly expressed in castration-resistant prostate cancer (CRPC) [19]. Furthermore, MALAT1 serves as an RNA cofactor of the polycomb protein enhancer of zeste homolog 2 (EZH2) and subsequently enhances oncogenesis in CRPC [20]. The expression of MALTA1 dramatically rises during the progression from hormone-sensitive to castration-resistant disease. Meanwhile, some studies have demonstrated that the silencing of MALAT1 leads to a metabolic reprogramming in prostate cancer [19-21].

Single-nucleotide polymorphisms (SNPs), i.e., variants of a single nucleotide occurring at a specific genomic position, have been demonstrated as genetic markers of both incidence and prognosis in prostate cancer [22,23]. SNPs in lncRNA could modulate the expression of lncRNA and subsequently alter the susceptibility to prostate cancer [24]. Although more and more studies have evaluated the regulatory pathway of MALAT1 in prostate cancer, scant data examining the association between MALAT1 SNPs and prostate cancer are available to date. Our study aimed to access the potential roles of MALAT1 polymorphisms in the clinicopathologic features of prostate cancer.

\section{Materials and Methods}

\subsection{Study Population}

From 2012 to 2017, a total of 579 consecutive patients with prostate adenocarcinoma was enrolled in this prospective study. The study was approved by the Institutional Review Board (IRB) of Taichung Veteran General Hospital (IRB No. CE19062A; 4 March 2019), and written informed consent was provided by each participant. These patients underwent robot-assisted radical prostatectomy with bilateral pelvic lymph node dissection. The medical profile included initial prostate-specific antigen (iPSA) when prostate cancer was diagnosed, clinical and pathologic tumor-node-metastasis (TNM) stage, pathologic Gleason grade group, D'Amico classification [25], and other pathologic findings.

The present study is an extension of our previous work exploring the predisposition of risk SNPs in prostate cancer and differentiating patients with extremely low oncologic risk from those traditionally defined as low risk in the D'Amico classification [25-27]. Thus, we divided the patients into three groups on the basis of their iPSA at diagnosis (iPSA $\leq 7 \mathrm{ng} / \mathrm{mL}$, iPSA $7-10 \mathrm{ng} / \mathrm{mL}$, and iPSA $>10 \mathrm{ng} / \mathrm{mL}$ ) to analyze the role of MALAT1 genotyping variation as a function of different levels of malignant potential in prostate cancer. 


\subsection{SNP Selection and DNA Extraction}

Three MALAT1 genetic variants, namely, rs3200401, rs619586, and rs1194338, were selected on the basis of data from the International HapMap Project dbSNP database and previous studies [28-30]. The allelic discrimination of MALAT1 polymorphisms was evaluated using an ABI StepOne Real-Time PCR System (Applied Biosystems, Foster City, CA, USA) and analyzed by TaqMan Assay with SDS 3.0 software (Applied Biosystems, Foster City, CA, USA), as described in our previous study [31].

Venous blood was collected from all participants before radical prostatectomy. Wholeblood samples were then placed in ethylenediaminetetraacetic acid (EDTA)-coated vacutainers and centrifuged for further DNA extraction. Isolations of DNA from the buffy coats were performed using QIAamp DNA Blood Mini Kits (Qiagen, Valencia, CA, USA), according to the manufacturer's protocol.

\subsection{Statistical Analysis}

Multiple logistic regression models were applied to access the adjusted odds ratio (AOR) with 95\% confidence interval (CI) between MALAT1 genotyping frequencies and three groups with different iPSA levels. The adjusted covariates included age at diagnosis, pathologic Gleason grade group, TNM stage, and some adverse pathologic features. A logistic regression model was used to estimate the odds ratio (OR) of each polymorphism in various clinical and pathologic presentations. Data were calculated using SAS statistical software (version 9.1; SAS Institute, Cary, NC, USA). The statistical significance was defined as a $p$-value $<0.05$.

\section{Results}

The distributions of clinical characteristics of enrolled subjects divided into three iPSA groups ( 153 cases with iPSA $\leq 7 \mathrm{ng} / \mathrm{mL}, 117$ cases with iPSA $7-10 \mathrm{ng} / \mathrm{mL}$, and 309 cases with iPSA $>10 \mathrm{ng} / \mathrm{mL}$ ) are demonstrated in Table 1 . There were higher incidences of Gleason grade $4+5$, advanced clinical and pathologic TNM stage, adverse pathologic features, and high-risk D'Amico classification among patients with iPSA $>10 \mathrm{ng} / \mathrm{mL}$ compared with the other two groups.

Table 1. Distributions of demographical characteristics in 579 patients with prostate cancer.

\begin{tabular}{|c|c|c|c|}
\hline \multirow{2}{*}{ Variable } & \multicolumn{3}{|c|}{ PSA at Diagnosis (ng/mL) } \\
\hline & $\leq 7(n=153)$ & $7-10(n=117)$ & $>10(n=309)$ \\
\hline \multicolumn{4}{|c|}{ Age at diagnosis (years) } \\
\hline$\leq 65$ & $71(46.4 \%)$ & $61(52.1 \%)$ & $113(36.6 \%)$ \\
\hline$>65$ & $82(53.6 \%)$ & $56(47.9 \%)$ & $196(63.4 \%)$ \\
\hline \multicolumn{4}{|c|}{ Pathologic Gleason grade group } \\
\hline $1+2$ & $123(80.4 \%)$ & $85(72.6 \%)$ & $152(49.2 \%)$ \\
\hline $3+4+5$ & $30(19.6 \%)$ & $32(27.4 \%)$ & $157(50.8 \%)$ \\
\hline \multicolumn{4}{|c|}{ Clinical T stage } \\
\hline $1+2$ & $144(94.1 \%)$ & $109(93.2 \%)$ & $248(80.3 \%)$ \\
\hline $3+4$ & $9(5.9 \%)$ & $8(6.8 \%)$ & $61(19.7 \%)$ \\
\hline \multicolumn{4}{|c|}{ Pathologic T stage } \\
\hline 2 & $114(74.5 \%)$ & $72(61.5 \%)$ & $120(38.8 \%)$ \\
\hline $3+4$ & $39(25.5 \%)$ & $45(38.5 \%)$ & $189(61.2 \%)$ \\
\hline \multicolumn{4}{|c|}{ Pathologic N stage } \\
\hline No & $146(95.4 \%)$ & $113(96.6 \%)$ & $384(87.7 \%)$ \\
\hline N1 & $7(4.6 \%)$ & $4(3.4 \%)$ & $38(12.3 \%)$ \\
\hline \multicolumn{4}{|c|}{ Extraprostatic extension } \\
\hline No & 107 (69.9\%) & $71(60.7 \%)$ & $148(47.9 \%)$ \\
\hline Yes & $46(30.1 \%)$ & $46(39.3 \%)$ & $161(52.1 \%)$ \\
\hline
\end{tabular}


Table 1. Cont.

\begin{tabular}{|c|c|c|c|}
\hline \multirow{2}{*}{ Variable } & \multicolumn{3}{|c|}{ PSA at Diagnosis (ng/mL) } \\
\hline & $\leq 7(n=153)$ & $7-10(n=117)$ & $>10(n=309)$ \\
\hline \multicolumn{4}{|l|}{ Seminal vesicle invasion } \\
\hline No & $145(94.8 \%)$ & $99(84.6 \%)$ & $208(67.3 \%)$ \\
\hline Yes & $8(5.2 \%)$ & $18(15.4 \%)$ & $101(32.7 \%)$ \\
\hline \multicolumn{4}{|l|}{ Perineural invasion } \\
\hline No & $53(34.6 \%)$ & $40(34.2 \%)$ & $62(20.1 \%)$ \\
\hline Yes & $100(65.4 \%)$ & $77(65.8 \%)$ & $247(79.9 \%)$ \\
\hline \multicolumn{4}{|l|}{ Lymphovascular invasion } \\
\hline No & $145(94.8 \%)$ & $101(86.3 \%)$ & $236(76.4 \%)$ \\
\hline Yes & $8(5.2 \%)$ & $16(13.7 \%)$ & $73(23.6 \%)$ \\
\hline \multicolumn{4}{|l|}{ D'Amico classification } \\
\hline Low/intermediate risk & $105(68.6 \%)$ & $85(72.6 \%)$ & $90(29.1 \%)$ \\
\hline High risk & $48(31.4 \%)$ & $32(27.4 \%)$ & $219(70.9 \%)$ \\
\hline \multicolumn{4}{|l|}{ Total score upgrade } \\
\hline No & $87(56.9 \%)$ & $74(63.2 \%)$ & $204(66.0 \%)$ \\
\hline Yes & $66(43.1 \%)$ & $43(36.8 \%)$ & $105(34.0 \%)$ \\
\hline \multicolumn{4}{|l|}{ Grade group upgrade } \\
\hline No & $84(54.9 \%)$ & $72(61.5 \%)$ & $187(60.5 \%)$ \\
\hline Yes & $69(45.1 \%)$ & $45(38.5 \%)$ & $122(39.5 \%)$ \\
\hline
\end{tabular}

Table 2 demonstrates the distribution frequencies of the three MALAT1 genotypes (namely, rs3200401, rs619586, and rs1194338) among 579 participants. The results showed that the percentage of patients carrying the homozygous polymorphic A allele in the rs1194338 polymorphism was significantly lower in both the iPSA 7-10 ng/mL group and the iPSA $>10 \mathrm{ng} / \mathrm{mL}$ group compared to patients in the iPSA $\leq 7 \mathrm{ng} / \mathrm{mL}$ group. The frequency remained significantly lower in at least one polymorphic $A$ allele $(C / A+A / A$ genotype $)$ at rs1194338 among participants with iPSA 7-10 ng/mL, although there was only a trend in participants with iPSA $>10 \mathrm{ng} / \mathrm{mL}$ of having lower percentages of $\mathrm{C} / \mathrm{A}+\mathrm{A} / \mathrm{A}$ genotype at the same loci. A nonsignificant higher frequency of A/G genotyping variant at rs619586 was also found in the iPSA $>10 \mathrm{ng} / \mathrm{mL}$ group compared to patients in the iPSA $\leq 7 \mathrm{ng} / \mathrm{mL}$ group. In contrast, there was no obvious trend in polymorphism frequencies at rs3200401.

Table 2. Distribution frequency of MALAT1 genotypes in 579 patients with prostate cancer.

\begin{tabular}{|c|c|c|c|c|c|}
\hline \multirow[t]{2}{*}{ Variable } & \multicolumn{5}{|c|}{ PSA at Diagnosis (ng/mL) } \\
\hline & $\leq 7(n=153)$ & $7-10(n=117)$ & $>10(n=309)$ & $\operatorname{AOR}(95 \% \mathrm{CI})^{\mathrm{a}}$ & $\operatorname{AOR}(95 \% \mathrm{CI})^{\mathrm{b}}$ \\
\hline \multicolumn{6}{|l|}{ rs3200401 } \\
\hline $\mathrm{CC}$ & $89(58.2 \%)$ & $76(65.0 \%)$ & $210(68.0 \%)$ & 1.00 & 1.00 \\
\hline $\mathrm{CT}$ & $57(37.3 \%)$ & $35(29.9 \%)$ & $89(28.8 \%)$ & $\begin{array}{l}0.760(0.440-1.315) \\
p=0.327\end{array}$ & $\begin{array}{l}0.754(0.464-1.224) \\
p=0.253\end{array}$ \\
\hline $\mathrm{TT}$ & $7(4.5 \%)$ & $6(5.1 \%)$ & $10(3.2 \%)$ & $\begin{array}{l}0.974(0.299-3.168) \\
p=0.965\end{array}$ & $\begin{array}{l}0.641(0.205-2.006) \\
p=0.445\end{array}$ \\
\hline $\mathrm{CT}+\mathrm{TT}$ & $64(41.8 \%)$ & $41(35.0 \%)$ & $99(32.0 \%)$ & $\begin{array}{l}0.785(0.465-1.326) \\
p=0.366\end{array}$ & $\begin{array}{l}0.741(0.464-1.183) \\
p=0.209\end{array}$ \\
\hline \multicolumn{6}{|l|}{ rs619586 } \\
\hline $\mathrm{AA}$ & $138(90.2 \%)$ & $105(89.7 \%)$ & $250(80.9 \%)$ & 1.00 & 1.00 \\
\hline AG & $15(9.8 \%)$ & $12(10.3 \%)$ & $59(19.1 \%)$ & $\begin{array}{l}1.154(0.506-2.630) \\
p=0.733\end{array}$ & $\begin{array}{l}1.942(0.972-3.881) \\
p=0.060\end{array}$ \\
\hline GG & $0(0 \%)$ & $0(0 \%)$ & $0(0 \%)$ & - & - \\
\hline $\mathrm{AG}+\mathrm{GG}$ & $15(9.8 \%)$ & $12(10.3 \%)$ & $59(19.1 \%)$ & $\begin{array}{l}1.154(0.506-2.630) \\
p=0.733\end{array}$ & $\begin{array}{l}1.942(0.972-3.881) \\
p=0.060\end{array}$ \\
\hline
\end{tabular}


Table 2. Cont.

\begin{tabular}{|c|c|c|c|c|c|}
\hline \multirow[t]{2}{*}{ Variable } & \multicolumn{5}{|c|}{ PSA at Diagnosis (ng/mL) } \\
\hline & $\leq 7(n=153)$ & $7-10(n=117)$ & $>10(n=309)$ & $\operatorname{AOR}(95 \% \mathrm{CI})^{a}$ & $\operatorname{AOR}(95 \% \mathrm{CI})^{b}$ \\
\hline \multicolumn{6}{|l|}{ rs1194338 } \\
\hline $\mathrm{CC}$ & $50(32.7 \%)$ & $55(47.0 \%)$ & $139(45.0 \%)$ & 1.00 & 1.00 \\
\hline CA & $70(45.8 \%)$ & $52(44.4 \%)$ & $128(41.4 \%)$ & $\begin{array}{l}0.703(0.406-1.218) \\
p=0.209\end{array}$ & $\begin{array}{l}0.758(0.457-1.256) \\
p=0.283\end{array}$ \\
\hline AA & $33(21.6 \%)$ & $10(8.5 \%)$ & $42(13.6 \%)$ & $\begin{array}{l}0.231(0.098-0.544) \\
p=0.001\end{array}$ & $\begin{array}{l}0.459(0.237-0.890) \\
p=0.021\end{array}$ \\
\hline $\mathrm{CA}+\mathrm{AA}$ & $103(67.3 \%)$ & $62(53.0 \%)$ & $170(55.0 \%)$ & $\begin{array}{l}0.545(0.324-0.916) \\
p=0.022\end{array}$ & $\begin{array}{l}0.661(0.412-1.060) \\
p=0.086\end{array}$ \\
\hline
\end{tabular}

The adjusted odds ratios (AORs) with their $95 \%$ confidence intervals (CIs) were estimated by multiple logistic regression models after controlling for age at diagnosis, pathologic Gleason grade group, clinical T stage, pathologic T stage, pathologic N stage, extraprostatic extension, seminal vesicle invasion, perineural invasion, lymphovascular invasion, and D'Amico classification. ${ }^{\text {a }}$ AORs with their $95 \%$ CIs were calculated between patients with PSA level $\leq 7 \mathrm{ng} / \mathrm{mL}$ and PSA level $7-10 \mathrm{ng} / \mathrm{mL} ;{ }^{b}$ AORs with their $95 \%$ CIs were calculated between patients with PSA level $\leq 7 \mathrm{ng} / \mathrm{mL}$, and PSA level $>10 \mathrm{ng} / \mathrm{mL}$.

Subsequent analysis of SNP variants at rs619586 and rs1194338 to evaluate their potential relationship with the clinicopathologic features was undertaken in not only all participants (Tables 3 and 4 ) but also focusing on patients with iPSA $>10 \mathrm{ng} / \mathrm{mL}$ (Table 5). Patients with a polymorphic $\mathrm{G}$ allele at rs619586 had a significantly higher risk of advanced Gleason grade (AOR 1.764, 95\% CI 1.011-3.077, $p=0.046$ ) (Table 3). For patients with at least one polymorphic A allele at rs1194338, the risk of pathologic lymph node invasion was significantly increased regardless of iPSA level (AOR 3.348, 95\% CI 1.501-7.469, $p=0.003$ ) (Table 4). This significant association between rs1194338 and node-positive disease was mainly observed in cases with iPSA $>10 \mathrm{ng} / \mathrm{mL}$ (AOR 3.452, 95\% CI 1.350-8.826, $p=0.010$ ) (Table 5). However, the presence of MALAT1 genotyping variants was not significantly associated with biochemical recurrence (BCR) and overall survival (OS) in the present study.

Table 3. Odds ratio (OR) and 95\% confidence interval (CI) of clinical status and MALAT1 rs619586 genotypic frequencies in 579 patients with prostate cancer.

\begin{tabular}{|c|c|c|c|c|}
\hline \multirow{2}{*}{$\begin{array}{c}\text { Variable } \\
\text { rs619586 }\end{array}$} & \multicolumn{4}{|c|}{ Genotypic Frequencies } \\
\hline & AA $(N=493)$ & $\mathrm{AG}+\mathrm{GG}(N=86)$ & AOR $(95 \% \mathrm{CI})$ & $p$-Value \\
\hline \multicolumn{5}{|c|}{ Pathologic Gleason grade group } \\
\hline $1+2$ & $317(64.3 \%)$ & $43(50.0 \%)$ & 1.00 & \multirow[t]{2}{*}{$p=0.046^{*}$} \\
\hline $3+4+5$ & $176(35.7 \%)$ & $43(50.0 \%)$ & $1.764(1.011-3.077)$ & \\
\hline \multicolumn{5}{|c|}{ Clinical $\mathrm{T}$ stage } \\
\hline $1+2$ & $426(86.4 \%)$ & $75(87.2 \%)$ & 1.00 & \multirow[t]{2}{*}{$p=0.605$} \\
\hline $3+4$ & $67(13.6 \%)$ & $11(12.8 \%)$ & $0.814(0.374-1.773)$ & \\
\hline \multicolumn{5}{|c|}{ Pathologic T stage } \\
\hline 2 & $265(53.8 \%)$ & $41(47.7 \%)$ & 1.00 & \multirow[t]{2}{*}{$p=0.748$} \\
\hline $3+4$ & $228(46.2 \%)$ & $45(52.3 \%)$ & $0.870(0.373-2.031)$ & \\
\hline \multicolumn{5}{|c|}{ Pathologic N stage } \\
\hline No & $447(90.7 \%)$ & $83(96.5 \%)$ & 1.00 & \multirow[t]{2}{*}{$p=0.086$} \\
\hline N1 & $46(9.3 \%)$ & $3(3.5 \%)$ & $0.389(0.125-1.324)$ & \\
\hline \multicolumn{5}{|c|}{ Extraprostatic extension } \\
\hline No & $280(56.8 \%)$ & $46(53.5 \%)$ & 1.00 & \multirow[t]{2}{*}{$p=0.838$} \\
\hline Yes & $213(43.2 \%)$ & $40(46.5 \%)$ & $0.923(0.427-1.996)$ & \\
\hline \multicolumn{5}{|c|}{ Seminal vesicle invasion } \\
\hline No & $389(78.9 \%)$ & $63(73.3 \%)$ & 1.00 & \multirow[t]{2}{*}{$p=0.504$} \\
\hline Yes & $104(21.1 \%)$ & $23(26.7 \%)$ & $1.276(0.625-2.606)$ & \\
\hline \multicolumn{5}{|c|}{ Perineural invasion } \\
\hline No & $138(28.0 \%)$ & $17(19.8 \%)$ & 1.00 & \multirow[t]{2}{*}{$p=0.339$} \\
\hline Yes & $355(72.0 \%)$ & $69(80.2 \%)$ & $1.363(0.722-2.573)$ & \\
\hline
\end{tabular}


Table 3. Cont

\begin{tabular}{|c|c|c|c|c|}
\hline \multirow{2}{*}{$\begin{array}{c}\text { Variable } \\
\text { rs619586 }\end{array}$} & \multicolumn{4}{|c|}{ Genotypic Frequencies } \\
\hline & AA $(N=493)$ & $\mathrm{AG}+\mathrm{GG}(N=86)$ & AOR $(95 \% \mathrm{CI})$ & $p$-Value \\
\hline \multicolumn{5}{|l|}{ Lymphovascular invasion } \\
\hline No & $412(83.6 \%)$ & $70(81.4 \%)$ & 1.00 & \multirow[t]{2}{*}{$p=0.604$} \\
\hline Yes & $81(16.4 \%)$ & $16(18.6 \%)$ & $1.210(0.590-2.481)$ & \\
\hline \multicolumn{5}{|l|}{ D'Amico classification } \\
\hline Low/intermediate risk & $246(49.9 \%)$ & $34(39.5 \%)$ & 1.00 & \multirow[t]{2}{*}{$p=0.104$} \\
\hline High risk & $247(50.1 \%)$ & $52(60.5 \%)$ & $1.486(0.878-2.516)$ & \\
\hline \multicolumn{5}{|l|}{ Total score upgrade } \\
\hline No & $313(63.5 \%)$ & $52(60.5 \%)$ & 1.00 & \multirow[t]{2}{*}{$p=0.931$} \\
\hline Yes & $180(36.5 \%)$ & $34(39.5 \%)$ & $1.041(0.413-2.628)$ & \\
\hline \multicolumn{5}{|l|}{ Grade group upgrade } \\
\hline No & $296(60.0 \%)$ & $47(54.7 \%)$ & 1.00 & \multirow[t]{2}{*}{$p=0.913$} \\
\hline Yes & $197(40.0 \%)$ & $39(45.3 \%)$ & $1.053(0.417-2.656)$ & \\
\hline
\end{tabular}

The adjusted odds ratios (AORs) with their $95 \%$ confidence intervals (CIs) were estimated by multiple logistic regression models after controlling for pathologic Gleason grade group, clinical T stage, pathologic $\mathrm{T}$ stage, pathologic $\mathrm{N}$ stage, extraprostatic extension, seminal vesicle invasion, perineural invasion, lymphovascular invasion, D'Amico classification, total score upgrade, and Grade group upgrade. ${ }^{*} p<0.05$ was considered statistically significant.

Table 4. Odds ratio (OR) and 95\% confidence interval (CI) of clinical status and MALAT1 rs1194338 genotypic frequencies in 579 patients with prostate cancer.

\begin{tabular}{|c|c|c|c|c|}
\hline \multirow{2}{*}{$\begin{array}{l}\text { Variable } \\
\text { rs1194338 }\end{array}$} & \multicolumn{4}{|c|}{ Genotypic Frequencies } \\
\hline & $\mathrm{CC}(N=244)$ & $\mathrm{CA}+\mathrm{AA}(N=335)$ & AOR $(95 \% \mathrm{CI})$ & $p$-Value \\
\hline \multicolumn{5}{|c|}{ Pathologic Gleason grade group } \\
\hline $1+2$ & $151(61.9 \%)$ & $209(62.4 \%)$ & 1.00 & \multirow[t]{2}{*}{$p=0.918$} \\
\hline $3+4+5$ & $93(38.1 \%)$ & $126(37.6 \%)$ & $0.978(0.644-1.487)$ & \\
\hline \multicolumn{5}{|l|}{ Clinical T stage } \\
\hline $1+2$ & $212(86.9 \%)$ & $289(86.3 \%)$ & 1.00 & \multirow[t]{2}{*}{$p=0.981$} \\
\hline $3+4$ & $32(13.1 \%)$ & $46(13.7 \%)$ & $0.993(0.561-1.757)$ & \\
\hline \multicolumn{5}{|l|}{ Pathologic T stage } \\
\hline 2 & $123(50.4 \%)$ & $183(54.6 \%)$ & 1.00 & \multirow{2}{*}{$p=0.340$} \\
\hline $3+4$ & $121(49.6 \%)$ & $152(45.4 \%)$ & $0.738(0.395-1.377)$ & \\
\hline \multicolumn{5}{|l|}{ Pathologic N stage } \\
\hline No & $232(95.1 \%)$ & $298(89.0 \%)$ & 1.00 & \multirow[t]{2}{*}{$p=0.003 *$} \\
\hline N1 & $12(4.9 \%)$ & $37(11.0 \%)$ & $3.348(1.501-7.469)$ & \\
\hline \multicolumn{5}{|l|}{ Extraprostatic extension } \\
\hline No & $138(56.6 \%)$ & $188(56.1 \%)$ & 1.00 & \multirow[t]{2}{*}{$p=0.189$} \\
\hline Yes & $106(43.4 \%)$ & $147(43.9 \%)$ & $1.479(0.825-2.651)$ & \\
\hline \multicolumn{5}{|l|}{ Seminal vesicle invasion } \\
\hline No & $188(77.0 \%)$ & $264(78.8 \%)$ & 1.00 & \multirow[t]{2}{*}{$p=0.749$} \\
\hline Yes & $56(23.0 \%)$ & $71(21.2 \%)$ & $0.915(0.532-1.575)$ & \\
\hline \multicolumn{5}{|l|}{ Perineural invasion } \\
\hline No & $53(21.7 \%)$ & $102(30.4 \%)$ & 1.00 & \multirow[t]{2}{*}{$p=0.028 *$} \\
\hline Yes & $191(78.3 \%)$ & $233(69.6 \%)$ & $0.614(0.397-0.949)$ & \\
\hline \multicolumn{5}{|l|}{ Lymphovascular invasion } \\
\hline No & $203(83.2 \%)$ & $279(83.3 \%)$ & 1.00 & \multirow[t]{2}{*}{$p=0.408$} \\
\hline Yes & $41(16.8 \%)$ & $56(16.7 \%)$ & $0.785(0.442-1.393)$ & \\
\hline \multicolumn{5}{|l|}{ D'Amico classification } \\
\hline Low/intermediate risk & $113(46.3 \%)$ & $167(49.9 \%)$ & 1.00 & \multirow[t]{2}{*}{$p=0.399$} \\
\hline High risk & $131(53.7 \%)$ & $168(50.1 \%)$ & $0.848(0.577-1.244)$ & \\
\hline \multicolumn{5}{|l|}{ Total score upgrade } \\
\hline No & $145(59.4 \%)$ & $220(65.7 \%)$ & 1.00 & \multirow[t]{2}{*}{$p=0.073$} \\
\hline Yes & $99(40.6 \%)$ & $115(34.3 \%)$ & $0.508(0.243-1.065)$ & \\
\hline
\end{tabular}


Table 4. Cont.

\begin{tabular}{lllll}
\hline Variable & \multicolumn{2}{l}{ Genotypic Frequencies } & & \\
\hline rs1194338 & CC $(\boldsymbol{N}=\mathbf{2 4 4})$ & CA + AA $(\boldsymbol{N}=\mathbf{3 3 5})$ & AOR (95\% CI) & $p$-Value \\
\hline Grade group upgrade & & & & \\
No & $140(57.4 \%)$ & $203(60.6 \%)$ & 1.00 & 0.188 \\
Yes & $104(42.6 \%)$ & $132(39.4 \%)$ & $1.642(0.784-3.437)$ & \\
\hline
\end{tabular}

The adjusted odds ratios (AORs) with their $95 \%$ confidence intervals (CIs) were estimated by multiple logistic regression models after controlling for pathologic Gleason grade group, clinical T stage, pathologic $\mathrm{T}$ stage, pathologic $\mathrm{N}$ stage, extraprostatic extension, seminal vesicle invasion, perineural invasion, lymphovascular invasion, D'Amico classification, total score upgrade, and Grade group upgrade. ${ }^{*} p<0.05$ was considered statistically significant.

Table 5. Odds ratio (OR) and 95\% confidence interval (CI) of clinical status and MALAT1 rs1194338 genotypic frequencies in 309 patients with prostate cancer with a PSA concentration $>10 \mathrm{ng} / \mathrm{mL}$.

\begin{tabular}{|c|c|c|c|c|}
\hline \multirow{2}{*}{$\begin{array}{l}\text { Variable } \\
\text { rs1194338 }\end{array}$} & \multicolumn{4}{|c|}{ Genotypic Frequencies } \\
\hline & $\mathrm{CC}(N=139)$ & $\mathrm{CA}+\mathrm{AA}(N=170)$ & AOR $(95 \% \mathrm{CI})$ & $p$-Value \\
\hline \multicolumn{5}{|c|}{ Pathologic Gleason grade group } \\
\hline $1+2$ & $69(49.6 \%)$ & $83(48.8 \%)$ & 1.00 & \multirow[t]{2}{*}{$p=0.943$} \\
\hline $3+4+5$ & $70(50.4 \%)$ & $87(51.2 \%)$ & $0.980(0.567-1.695)$ & \\
\hline \multicolumn{5}{|l|}{ Clinical T stage } \\
\hline $1+2$ & $111(79.9 \%)$ & $137(80.6 \%)$ & 1.00 & \multirow[t]{2}{*}{$p=0.594$} \\
\hline $3+4$ & $28(20.1 \%)$ & $33(19.4 \%)$ & $0.835(0.430-1.622)$ & \\
\hline \multicolumn{5}{|l|}{ Pathologic T stage } \\
\hline 2 & $54(38.8 \%)$ & $66(38.8 \%)$ & 1.00 & \multirow[t]{2}{*}{$p=0.920$} \\
\hline $3+4$ & $85(61.2 \%)$ & $104(61.2 \%)$ & $0.955(0.388-2.351)$ & \\
\hline \multicolumn{5}{|l|}{ Pathologic N stage } \\
\hline No & $129(92.8 \%)$ & $142(83.5 \%)$ & 1.00 & \multirow[t]{2}{*}{$p=0.010 *$} \\
\hline $\mathrm{N} 1$ & $10(7.2 \%)$ & $28(16.5 \%)$ & $3.452(1.350-8.826)$ & \\
\hline \multicolumn{5}{|l|}{ Extraprostatic extension } \\
\hline No & $69(49.6 \%)$ & $79(46.5 \%)$ & 1.00 & \multirow[t]{2}{*}{$p=0.393$} \\
\hline Yes & $70(50.4 \%)$ & $91(53.5 \%)$ & $1.437(0.625-3.302)$ & \\
\hline \multicolumn{5}{|l|}{ Seminal vesicle invasion } \\
\hline No & $94(67.6 \%)$ & $114(67.1 \%)$ & 1.00 & \multirow[t]{2}{*}{$p=0.992$} \\
\hline Yes & $45(32.4 \%)$ & $56(32.9 \%)$ & $1.003(0.531-1.897)$ & \\
\hline \multicolumn{5}{|l|}{ Perineural invasion } \\
\hline No & $22(15.8 \%)$ & $40(23.5 \%)$ & 1.00 & \multirow[t]{2}{*}{$p=0.057$} \\
\hline Yes & $117(84.2 \%)$ & $130(76.5 \%)$ & $0.501(0.254-1.012)$ & \\
\hline \multicolumn{5}{|l|}{ Lymphovascular invasion } \\
\hline No & $107(77.0 \%)$ & $129(75.9 \%)$ & 1.00 & \multirow[t]{2}{*}{$p=0.354$} \\
\hline Yes & $32(23.0 \%)$ & $41(24.1 \%)$ & $0.722(0.363-1.438)$ & \\
\hline \multicolumn{5}{|l|}{ D'Amico classification } \\
\hline Low/intermediate risk & $38(27.3 \%)$ & $52(30.6 \%)$ & 1.00 & \multirow[t]{2}{*}{$p=0.473$} \\
\hline High risk & $101(72.7 \%)$ & $118(69.4 \%)$ & $0.815(0.466-1.425)$ & \\
\hline \multicolumn{5}{|l|}{ Total score upgrade } \\
\hline No & $87(62.6 \%)$ & $117(68.8 \%)$ & 1.00 & \multirow[t]{2}{*}{$p=0.203$} \\
\hline Yes & $52(37.4 \%)$ & $53(31.2 \%)$ & $0.552(0.221-1.378)$ & \\
\hline \multicolumn{5}{|l|}{ Grade group upgrade } \\
\hline No & $82(59.0 \%)$ & $105(61.8 \%)$ & 1.00 & \multirow[t]{2}{*}{$p=0.322$} \\
\hline Yes & $57(41.0 \%)$ & $65(38.2 \%)$ & $1.582(0.639-3.916)$ & \\
\hline
\end{tabular}

The adjusted odds ratios (AORs) with their $95 \%$ confidence intervals (CIs) were estimated by multiple logistic regression models after controlling for pathologic Gleason grade group, clinical T stage, pathologic $\mathrm{T}$ stage, pathologic N stage, extraprostatic extension, seminal vesicle invasion, perineural invasion, lymphovascular invasion, D'Amico classification, total score upgrade, and Grade group upgrade. ${ }^{*} p<0.05$ was considered statistically significant.

\section{Discussion}

This is the first study to assess MALAT1 polymorphisms and their clinicopathologic impact on Taiwanese men with operatable prostate cancer. Our results indicated that MALAT1 SNP rs619586 is positively associated with advanced Gleason grade, while rs1194338 plays a part in lymph node metastasis, especially in patients with iPSA $>10 \mathrm{ng} / \mathrm{mL}$. Because 
MALAT1 is considered an oncogene in genitourinary cancer [32], we believed that MALAT1 genotyping variants might enhance its regulatory functions in prostate cancer, being subsequently responsible for cell proliferation and tumor invasion.

Lymph node invasion, one form of advanced prostate cancer, has a strong influence on adverse prognosis in either distant metastasis or cancer-specific survival [33,34]. Moreover, the number of positive lymph nodes can act as a powerful predictor for both biochemical recurrence and cancer-related death $[35,36]$. Thus, adjuvant hormonal therapy with/without radiotherapy is advocated for nodal metastasis prostate cancer in order to eliminate the occult micrometastasis, as well as improve cancer-specific and overall survival, especially in patients with high-risk prostate cancer [6,37-40].

However, there are no definite preoperative diagnosing tools to confirm nodal invasion. Traditional imaging tools including conventional computed tomography (CT) and magnetic resonance $(\mathrm{MR})$ imaging are insensitive and inaccurate when the sizes of metastatic nodes are not prominent enough. Only some lymph node prediction nomograms are available today $[41,42]$. The current urologic guidelines suggest that various nomograms could be helpful in decision making for concurrent pelvic lymph node dissection during radical prostatectomy $[11,43]$. The parameters involved in these nomograms include age, iPSA, clinical stage, primary/secondary Gleason score, and the percentage of positive cores [42]. Unfortunately, there are still limitations to these nomograms, and further validation is persistently required [44-47].

Currently, pelvic lymph node dissection remains the gold standard for lymph node staging [6,7], while extended pelvic lymph node dissection is recommended for the correct staging in contrast to limited pelvic lymphadenectomy [48]. However, this invasive procedure could lead to various complications, although it provides both diagnostic and therapeutic benefits in intermediate-risk and high-risk prostate cancer [8-10]. Therefore, more meticulous but less invasive predictors are required to evaluate either the possibility of nodal metastasis or the indication for lymph node dissection.

Genetic polymorphisms could help the risk predictions of prostate cancer and might also interfere with oncologic prognosis in prostate cancer [22,49]. Our previous research showed that carbonic anhydrate 9 (CA9) polymorphism is associated with a 4.5-fold increased risk of lymph node metastasis while growth arrest-specific 5 (GAS5) SNPs play a protective role in nodal invasion (OR $0.545, p=0.043$ ) [50,51]. The present study demonstrates that MALAT1 polymorphism rs1194338 leads to a 3.348-fold increased risk of node-positive prostate cancer. The susceptibility to node-positive prostate cancer was more remarkable in the PSA $>10 \mathrm{ng} / \mathrm{mL}$ group (Table 5), although fewer patients in the PSA $>10 \mathrm{ng} / \mathrm{mL}$ group carried at least one polymorphic A allele at rs1194338 compared to patients with a PSA level lower than $10 \mathrm{ng} / \mathrm{mL}$ (Table 2). With more information about how genotyping variants interfere with lymph node metastasis, we could develop an innovative lymph node nomogram in the future by taking account of the status of these SNPs to evaluate the indication of lymph node dissection and further requirement for adjuvant therapy.

The roles of MALAT1 in tumorigenesis are complicated, as it can function as either a promoter or a suppressor in metastasis depending on the mechanism of action in different cancers [52]. Polymorphisms of MALAT1 could alter its regulatory roles in the presplicing process and gene expression. Previous studies found that rs619586 A > G polymorphisms have a protective effect toward papillary thyroid cancer $(\mathrm{OR}=0.76,95 \% \mathrm{CI}=0.60-0.95$, $p=0.017)$ and hepatocellular carcinoma (AOR $=0.29,95 \% \mathrm{CI}=0.11-0.77, p=0.013)[31,53]$. Another MALAT1 SNP rs1194338 was also identified as a protective factor toward colorectal cancer susceptibility $(\mathrm{OR}=0.70,95 \% \mathrm{CI}=0.49-0.99, p=0.045)$ [54]. In stark contrast, these two SNPs were both associated with an aggressive tumorigenesis of prostate cancer in this cohort. Our results suggest that the regulatory pathway of MALAT1 SNPs in prostate cancer might be different from other malignancies.

There were some limitations in the present study. Firstly, we only recruited patients who underwent radical prostatectomy. They were relatively younger with a mean age of 67.1 years old and with better functional performance, which allowed them to receive gen- 
eral anesthesia. These patients also had a less advanced and more operatable disease. These baseline characteristics might have served as protective confounders for prognosis and led to an insignificant result in biochemical recurrence (BCR), progression-free survival (PFS), and overall survival (OS). Secondly, the participants were assumed to have a hormonesensitive status since they were treatment-naïve before prostatectomy. Therefore, this study only demonstrates the predictive impact of MALAT1 SNPs in men with hormone-sensitive prostate cancer (HSPC). Compared to operatable HSPC, castration-resistant prostate cancer (CRPC) is a more heterogenous disease and presents with a different cell surface protein profile, which may alter the regulatory function of lncRNAs in oncogenesis. Further research assessing the potential effects of MALAT1 SNPs on the castration process and therapeutic response will be conducted in the future. Thirdly, the follow-up period did not exceed 10 years, whereas life expectancy after the diagnosis of prostate cancer is 9.7 (95\% CI 9.5-9.8) years in Taiwan [55]. Since prostate cancer is a relatively slow-progressing disease compared to other aggressive malignancies, this insufficient follow-up time might have led to the lack of difference in BCR, PFS, and OS. Furthermore, only Taiwanese men were accessed in this single-center study with a relatively small sample size. Hence, further research on larger cohorts and even the international multi-centers to confirm the association between these MALAT1 SNPs and clinicopathological characteristics patients with prostate cancer needs to be conducted.

A previous meta-analysis concluded that the overexpression of MALAT1 could predict lymph node metastasis in various types of cancer (pooled OR $=2.34, \mathrm{CI}=1.61-3.40$, $p<0.001$ ) [56]. However, only blood samples were collected in this cohort, and no cancer tissue was concomitantly harvested from the prostate specimen. Thus, we could only identify the relationship between MALAT1 genotyping frequency and clinicopathologic characteristics. Further research is still required to confirm the definite associations between MALAT1 expression and oncologic outcome.

\section{Conclusions}

MALAT1 SNPs are positively associated with adverse pathologic features including nodal metastasis and advanced Gleason grades in operatable HSPC. With more information about risk SNPs in node-positive prostate cancer, a modified lymph node nomogram can be developed to enhance preoperative predictions and facilitate surgical plans in the era of precision medicine. Further studies examining the potential roles of lncRNA MALAT1 in CRPC are also needed.

Author Contributions: Conceptualization, J.-C.H., C.-Y.L. and S.-F.Y.; methodology, J.-C.H., Y.-E.C. and S.-F.Y.; formal analysis, S.-F.Y.; resources, J.-C.H., S.-S.W., K.-Y.C., J.-R.L., C.-S.C., S.-C.H., C.-K.Y., Y.-C.O., C.-L.C. and C.-Y.L.; writing-original draft preparation, J.-C.H., C.-Y.L. and S.-F.Y.; writingreview and editing, J.-C.H. and S.-F.Y. All authors have read and agreed to the published version of the manuscript.

Funding: This research was funded by Taichung Veteran General Hospital, grant number TCVGH1105001B.

Institutional Review Board Statement: This study was conducted according to the guidelines of the Declaration of Helsinki and approved by the Institutional Review Board of Taichung Veteran General Hospital (IRB No. CE19062A; 4 March 2019).

Informed Consent Statement: Informed consent was obtained from all subjects involved in the study.

Data Availability Statement: The datasets generated for this study are available on request to the corresponding authors.

Conflicts of Interest: The authors declare no conflict of interest. 


\section{References}

1. Siegel, R.L.; Miller, K.D.; Fuchs, H.E.; Jemal, A. Cancer statistics, 2021. CA Cancer J. Clin. 2021, 71, 7-33. [CrossRef]

2. Negoita, S.; Feuer, E.J.; Mariotto, A.; Cronin, K.A.; Petkov, V.I.; Hussey, S.K.; Benard, V.; Henley, S.J.; Anderson, R.N.; Fedewa, S.; et al. Annual report to the nation on the status of cancer, part ii: Recent changes in prostate cancer trends and disease characteristics. Cancer 2018, 124, 2801-2814. [CrossRef] [PubMed]

3. Zhou, C.K.; Check, D.P.; Lortet-Tieulent, J.; Laversanne, M.; Jemal, A.; Ferlay, J.; Bray, F.; Cook, M.B.; Devesa, S.S. Prostate cancer incidence in 43 populations worldwide: An analysis of time trends overall and by age group. Int. J. Cancer 2016, 138, 1388-1400. [CrossRef]

4. Annual Report. 2018: Taiwan Cancer Registry; Health Promotion Administration, Ministry of Health and Welfare. Available online: https://www.Hpa.Gov.Tw / pages/ashx/file.Ashx?Filepath= \{\}/file/attach/13498/file_15611.Pdf (accessed on 20 July 2021).

5. Shih, H.J.; Fang, S.C.; An, L.; Shao, Y.J. Early-onset prostate cancer is associated with increased risks of disease progression and cancer-specific mortality. Prostate 2021, 81, 118-126. [CrossRef]

6. Sankineni, S.; Brown, A.M.; Fascelli, M.; Law, Y.M.; Pinto, P.A.; Choyke, P.L.; Turkbey, B. Lymph node staging in prostate cancer. Curr. Urol. Rep. 2015, 16, 30. [CrossRef] [PubMed]

7. Thoeny, H.C.; Barbieri, S.; Froehlich, J.M.; Turkbey, B.; Choyke, P.L. Functional and targeted lymph node imaging in prostate cancer: Current status and future challenges. Radiology 2017, 285, 728-743. [CrossRef] [PubMed]

8. Fossati, N.; Willemse, P.M.; Van den Broeck, T.; van den Bergh, R.C.N.; Yuan, C.Y.; Briers, E.; Bellmunt, J.; Bolla, M.; Cornford, P.; De Santis, M.; et al. The benefits and harms of different extents of lymph node dissection during radical prostatectomy for prostate cancer: A systematic review. Eur. Urol. 2017, 72, 84-109. [CrossRef]

9. Choo, M.S.; Kim, M.; Ku, J.H.; Kwak, C.; Kim, H.H.; Jeong, C.W. Extended versus standard pelvic lymph node dissection in radical prostatectomy on oncological and functional outcomes: A systematic review and meta-analysis. Ann. Surg. Oncol. 2017, 24, 2047-2054. [CrossRef] [PubMed]

10. Briganti, A.; Chun, F.K.; Salonia, A.; Suardi, N.; Gallina, A.; Da Pozzo, L.F.; Roscigno, M.; Zanni, G.; Valiquette, L.; Rigatti, P.; et al. Complications and other surgical outcomes associated with extended pelvic lymphadenectomy in men with localized prostate cancer. Eur. Urol. 2006, 50, 1006-1013. [CrossRef]

11. National Comprehensive Cancer Network (nccn) Clinical Proctice Guidelines in Prostate Cancer. Available online: https: //www.Nccn.Org/professionals/physician_gls/pdf/prostate.Pdf (accessed on 20 July 2021).

12. de Bono, J.; Mateo, J.; Fizazi, K.; Saad, F.; Shore, N.; Sandhu, S.; Chi, K.N.; Sartor, O.; Agarwal, N.; Olmos, D.; et al. Olaparib for metastatic castration-resistant prostate cancer. N. Engl. J. Med. 2020, 382, 2091-2102. [CrossRef]

13. Qian, Y.; Shi, L.; Luo, Z. Long non-coding rnas in cancer: Implications for diagnosis, prognosis, and therapy. Front. Med. 2020, 7, 612393. [CrossRef]

14. Ramnarine, V.R.; Kobelev, M.; Gibb, E.A.; Nouri, M.; Lin, D.; Wang, Y.; Buttyan, R.; Davicioni, E.; Zoubeidi, A.; Collins, C.C. The evolution of long noncoding rna acceptance in prostate cancer initiation, progression, and its clinical utility in disease management. Eur. Urol. 2019, 76, 546-559. [CrossRef]

15. Bradley, L.A.; Palomaki, G.E.; Gutman, S.; Samson, D.; Aronson, N. Comparative effectiveness review: Prostate cancer antigen 3 testing for the diagnosis and management of prostate cancer. J. Urol. 2013, 190, 389-398. [CrossRef]

16. Arriaga-Canon, C.; De La Rosa-Velázquez, I.A.; González-Barrios, R.; Montiel-Manríquez, R.; Oliva-Rico, D.; Jiménez-Trejo, F.; Cortés-González, C.; Herrera, L.A. The use of long non-coding rnas as prognostic biomarkers and therapeutic targets in prostate cancer. Oncotarget 2018, 9, 20872-20890. [CrossRef]

17. Goyal, B.; Yadav, S.R.M.; Awasthee, N.; Gupta, S.; Kunnumakkara, A.B.; Gupta, S.C. Diagnostic, prognostic, and therapeutic significance of long non-coding rna malat1 in cancer. Biochim. Biophys. Acta Rev. Cancer 2021, 1875, 188502. [CrossRef]

18. Wang, F.; Ren, S.; Chen, R.; Lu, J.; Shi, X.; Zhu, Y.; Zhang, W.; Jing, T.; Zhang, C.; Shen, J.; et al. Development and prospective multicenter evaluation of the long noncoding rna malat-1 as a diagnostic urinary biomarker for prostate cancer. Oncotarget 2014, 5, 11091-11102. [CrossRef] [PubMed]

19. Wang, R.; Sun, Y.; Li, L.; Niu, Y.; Lin, W.; Lin, C.; Antonarakis, E.S.; Luo, J.; Yeh, S.; Chang, C. Preclinical study using malat1 small interfering rna or androgen receptor splicing variant 7 degradation enhancer asc-j $9((\mathrm{r}))$ to suppress enzalutamide-resistant prostate cancer progression. Eur. Uro.l 2017, 72, 835-844. [CrossRef] [PubMed]

20. Wang, D.; Ding, L.; Wang, L.; Zhao, Y.; Sun, Z.; Karnes, R.J.; Zhang, J.; Huang, H. Lncrna malat1 enhances oncogenic activities of ezh2 in castration-resistant prostate cancer. Oncotarget 2015, 6, 41045-41055. [CrossRef] [PubMed]

21. Nanni, S.; Aiello, A.; Salis, C.; Re, A.; Cencioni, C.; Bacci, L.; Pierconti, F.; Pinto, F.; Ripoli, C.; Ostano, P.; et al. Metabolic reprogramming by malat1 depletion in prostate cancer. Cancers 2020, 13, 15. [CrossRef]

22. Zheng, S.L.; Sun, J.; Wiklund, F.; Smith, S.; Stattin, P.; Li, G.; Adami, H.O.; Hsu, F.C.; Zhu, Y.; Balter, K.; et al. Cumulative association of five genetic variants with prostate cancer. N. Engl. J. Med. 2008, 358, 910-919. [CrossRef]

23. Shui, I.M.; Lindstrom, S.; Kibel, A.S.; Berndt, S.I.; Campa, D.; Gerke, T.; Penney, K.L.; Albanes, D.; Berg, C.; Bueno-de-Mesquita, H.B.; et al. Prostate cancer (pca) risk variants and risk of fatal pca in the national cancer institute breast and prostate cancer cohort consortium. Eur. Urol. 2014, 65, 1069-1075. [CrossRef]

24. Guo, H.; Ahmed, M.; Zhang, F.; Yao, C.Q.; Li, S.; Liang, Y.; Hua, J.; Soares, F.; Sun, Y.; Langstein, J.; et al. Modulation of long noncoding rnas by risk snps underlying genetic predispositions to prostate cancer. Nat. Genet. 2016, 48, 1142-1150. [CrossRef] 
25. D'Amico, A.V.; Whittington, R.; Malkowicz, S.B.; Schultz, D.; Blank, K.; Broderick, G.A.; Tomaszewski, J.E.; Renshaw, A.A.; Kaplan, I.; Beard, C.J.; et al. Biochemical outcome after radical prostatectomy, external beam radiation therapy, or interstitial radiation therapy for clinically localized prostate cancer. JAMA 1998, 280, 969-974. [CrossRef]

26. Elshafei, A.; Li, Y.H.; Hatem, A.; Moussa, A.S.; Ethan, V.; Krishnan, N.; Li, J.; Jones, J.S. The utility of psa velocity in prediction of prostate cancer and high grade cancer after an initially negative prostate biopsy. Prostate 2013, 73, 1796-1802. [CrossRef] [PubMed]

27. Hu, J.C.; Lin, C.Y.; Wang, S.S.; Chiu, K.Y.; Li, J.R.; Chen, C.S.; Hung, S.C.; Yang, C.K.; Ou, Y.C.; Cheng, C.L.; et al. Impact of h19 polymorphisms on prostate cancer clinicopathologic characteristics. Diagnostics 2020, 10, 656. [CrossRef] [PubMed]

28. Zou, H.; Wu, L.X.; Tan, L.; Shang, F.F.; Zhou, H.H. Significance of single-nucleotide variants in long intergenic non-protein coding rnas. Front. Cell Dev. Biol. 2020, 8, 347. [CrossRef]

29. Wang, J.Z.; Xiang, J.J.; Wu, L.G.; Bai, Y.S.; Chen, Z.W.; Yin, X.Q.; Wang, Q.; Guo, W.H.; Peng, Y.; Guo, H.; et al. A genetic variant in long non-coding rna malat1 associated with survival outcome among patients with advanced lung adenocarcinoma: A survival cohort analysis. BMC Cancer 2017, 17, 167. [CrossRef]

30. Huang, X.; Zhang, W.; Shao, Z. Association between long non-coding rna polymorphisms and cancer risk: A meta-analysis. Biosci. Rep. 2018, 38, BSR20180365. [CrossRef]

31. Yuan, L.T.; Chang, J.H.; Lee, H.L.; Yang, Y.C.; Su, S.C.; Lin, C.L.; Yang, S.F.; Chien, M.H. Genetic variants of lncrna malat1 exert diverse impacts on the risk and clinicopathologic characteristics of patients with hepatocellular carcinoma. J. Clin. Med. 2019, 8, 1406. [CrossRef] [PubMed]

32. Flippot, R.; Beinse, G.; Boileve, A.; Vibert, J.; Malouf, G.G. Long non-coding rnas in genitourinary malignancies: A whole new world. Nat. Rev. Urol. 2019, 16, 484-504. [CrossRef]

33. Gervasi, L.A.; Mata, J.; Easley, J.D.; Wilbanks, J.H.; Seale-Hawkins, C.; Carlton, C.E., Jr.; Scardino, P.T. Prognostic significance of lymph nodal metastases in prostate cancer. J. Urol. 1989, 142, 332-336. [CrossRef]

34. Boorjian, S.A.; Thompson, R.H.; Siddiqui, S.; Bagniewski, S.; Bergstralh, E.J.; Karnes, R.J.; Frank, I.; Blute, M.L. Long-term outcome after radical prostatectomy for patients with lymph node positive prostate cancer in the prostate specific antigen era. J. Urol. 2007, 178, 864-870. [CrossRef]

35. Carlsson, S.V.; Tafe, L.J.; Chade, D.C.; Sjoberg, D.D.; Passoni, N.; Shariat, S.F.; Eastham, J.; Scardino, P.T.; Fine, S.W.; Touijer, K.A. Pathological features of lymph node metastasis for predicting biochemical recurrence after radical prostatectomy for prostate cancer. J. Urol. 2013, 189, 1314-1318. [CrossRef] [PubMed]

36. Mandel, P.; Kriegmair, M.C.; Bogdan, K.; Boehm, K.; Budaus, L.; Graefen, M.; Huland, H.; Tilki, D. Association between lymph node counts and oncological outcomes in lymph node positive prostate cancer. Eur. Urol. Focus 2017, 3, 248-255. [CrossRef]

37. Preisser, F.; Bandini, M.; Marchioni, M.; Nazzani, S.; Tian, Z.; Pompe, R.S.; Fossati, N.; Briganti, A.; Saad, F.; Shariat, S.F.; et al. Extent of lymph node dissection improves survival in prostate cancer patients treated with radical prostatectomy without lymph node invasion. Prostate 2018, 78, 469-475. [CrossRef] [PubMed]

38. Abdollah, F.; Gandaglia, G.; Suardi, N.; Capitanio, U.; Salonia, A.; Nini, A.; Moschini, M.; Sun, M.; Karakiewicz, P.I.; Shariat, S.F.; et al. More extensive pelvic lymph node dissection improves survival in patients with node-positive prostate cancer. Eur. Urol. 2015, 67, 212-219. [CrossRef]

39. Messing, E.M.; Manola, J.; Yao, J.; Kiernan, M.; Crawford, D.; Wilding, G.; di'SantAgnese, P.A.; Trump, D. Immediate versus deferred androgen deprivation treatment in patients with node-positive prostate cancer after radical prostatectomy and pelvic lymphadenectomy. Lancet Oncol. 2006, 7, 472-479, discussion 870-861. [CrossRef]

40. Gupta, M.; Patel, H.D.; Schwen, Z.R.; Tran, P.T.; Partin, A.W. Adjuvant radiation with androgen-deprivation therapy for men with lymph node metastases after radical prostatectomy: Identifying men who benefit. BJU Int. 2019, 123, 252-260. [CrossRef]

41. Briganti, A.; Larcher, A.; Abdollah, F.; Capitanio, U.; Gallina, A.; Suardi, N.; Bianchi, M.; Sun, M.; Freschi, M.; Salonia, A.; et al. Updated nomogram predicting lymph node invasion in patients with prostate cancer undergoing extended pelvic lymph node dissection: The essential importance of percentage of positive cores. Eur. Urol. 2012, 61, 480-487. [CrossRef] [PubMed]

42. Cimino, S.; Reale, G.; Castelli, T.; Favilla, V.; Giardina, R.; Russo, G.I.; Privitera, S.; Morgia, G. Comparison between briganti, partin and mskcc tools in predicting positive lymph nodes in prostate cancer: A systematic review and meta-analysis. Scand. J. Urol. 2017, 51, 345-350. [CrossRef]

43. European Assoication of Urology (eau) Guidelines for Prostate Cancer. Available online: https://uroweb.Org/guideline/ prostate-cancer/ (accessed on 15 July 2021).

44. Grivas, N.; Wit, E.; Tillier, C.; van Muilekom, E.; Pos, F.; Winter, A.; van der Poel, H. Validation and head-to-head comparison of three nomograms predicting probability of lymph node invasion of prostate cancer in patients undergoing extended and/or sentinel lymph node dissection. Eur J. Nucl Med. Mol. Imaging 2017, 44, 2213-2226. [CrossRef] [PubMed]

45. Caras, R.J.; Sterbis, J.R. Prostate cancer nomograms: A review of their use in cancer detection and treatment. Curr. Urol. Rep. 2014, 15, 391. [CrossRef] [PubMed]

46. Leyh-Bannurah, S.R.; Budaus, L.; Pompe, R.; Zaffuto, E.; Briganti, A.; Abdollah, F.; Montorsi, F.; Schiffmann, J.; Menon, M.; Shariat, S.F.; et al. North american population-based validation of the national comprehensive cancer network practice guideline recommendation of pelvic lymphadenectomy in contemporary prostate cancer. Prostate 2017, 77, 542-548. [CrossRef] [PubMed] 
47. Venclovas, Z.; Muilwijk, T.; Matjosaitis, A.J.; Jievaltas, M.; Joniau, S.; Milonas, D. Head-to-head comparison of two nomograms predicting probability of lymph node invasion in prostate cancer and the therapeutic impact of higher nomogram threshold. $J$. Clin. Med. 2021, 10, 999. [CrossRef]

48. Mattei, A.; Fuechsel, F.G.; Bhatta Dhar, N.; Warncke, S.H.; Thalmann, G.N.; Krause, T.; Studer, U.E. The template of the primary lymphatic landing sites of the prostate should be revisited: Results of a multimodality mapping study. Eur. Urol. 2008, 53, 118-125. [CrossRef] [PubMed]

49. Morote, J.; Del Amo, J.; Borque, A.; Ars, E.; Hernandez, C.; Herranz, F.; Arruza, A.; Llarena, R.; Planas, J.; Viso, M.J.; et al. Improved prediction of biochemical recurrence after radical prostatectomy by genetic polymorphisms. J. Urol. 2010, 184, 506-511. [CrossRef] [PubMed]

50. Lin, C.Y.; Wang, S.S.; Yang, C.K.; Li, J.R.; Chen, C.S.; Hung, S.C.; Chiu, K.Y.; Cheng, C.L.; Ou, Y.C.; Yang, S.F. Genetic polymorphism and carbonic anhydrase 9 expression can predict nodal metastatic prostate cancer risk in patients with prostate-specific antigen levels $\leq 10 \mathrm{ng} / \mathrm{ml}$ at initial biopsy. Urol. Oncol. 2019, 37, 814.e9-814.e16. [CrossRef]

51. Lin, C.Y.; Wang, S.S.; Yang, C.K.; Li, J.R.; Chen, C.S.; Hung, S.C.; Chiu, K.Y.; Cheng, C.L.; Ou, Y.C.; Yang, S.F. Impact of gas5 genetic polymorphism on prostate cancer susceptibility and clinicopathologic characteristics. Int. J. Med. Sci. 2019, 16, 1424-1429. [CrossRef]

52. Sun, Y.; Ma, L. New insights into long non-coding rna malat1 in cancer and metastasis. Cancers 2019, 11, 216. [CrossRef]

53. Wen, J.; Chen, L.; Tian, H.; Li, J.; Zhang, M.; Cao, Q.; Zhang, W.; Chen, S.; Shi, L. Effect of malat1 polymorphisms on papillary thyroid cancer in a chinese population. J. Cancer 2019, 10, 5714-5721. [CrossRef]

54. Li, Y.; Bao, C.; Gu, S.; Ye, D.; Jing, F.; Fan, C.; Jin, M.; Chen, K. Associations between novel genetic variants in the promoter region of malat1 and risk of colorectal cancer. Oncotarget 2017, 8, 92604-92614. [CrossRef] [PubMed]

55. Wu, T.Y.; Chung, C.H.; Lin, C.N.; Hwang, J.S.; Wang, J.D. Lifetime risks, loss of life expectancy, and health care expenditures for 19 types of cancer in taiwan. Clin. Epidemiol. 2018, 10, 581-591. [CrossRef] [PubMed]

56. Li, J.; Cui, Z.; Li, H.; Lv, X.; Gao, M.; Yang, Z.; Bi, Y.; Zhang, Z.; Wang, S.; Zhou, B.; et al. Clinicopathological and prognostic significance of long noncoding rna malat1 in human cancers: A review and meta-analysis. Cancer Cell Int. 2018, 18, 109. [CrossRef] [PubMed] 